\title{
El policentrismo en Chile: medición exploratoria para el sistema de ciudades de las regiones de La Araucanía, de Los Ríos y de Los Lagos ${ }^{1}$
}

\author{
Francisco Ramón Maturana Miranda² y Federico Arenas Vásquez ${ }^{3}$
}

\begin{abstract}
RESUMEN
El policentrismo es un concepto que ha tomado fuerzas en los últimos años como un objetivo de la política territorial europea, cuyo fin es generar espacios más equilibrados y cohesionados. Su definición y medición ha generado un amplio debate en los medios científicos internacionales en el cual Chile ha estado rezagado. Así, esta publicación busca identificar el concepto de policentrismo, sus vertientes y proponer algunas mejoras a los métodos de medición que se han utilizado. Para tal objetivo se aplicó una medición teórica del nivel de policentrismo de tres regiones del sur de Chile, La Araucanía, Los Ríos y Los Lagos, cuyos resultados sugieren la existencia de un medio bajo policentrismo como sistema en su conjunto y un bajo nivel de policentrismo en cada región.
\end{abstract}

Palabras clave: Policentrismo, sistemas de ciudades, Chile.

\begin{abstract}
Polycentricity is a concept that has gained strength in recent years as one of the European territorial policy strategic objectives, which aims to promote more balanced and cohesive spaces. Its definition and measurement has generated much debate in the international scientific community, in which Chile has been lagging. This publication aims to define the concept of polycentricity, its main aspects and to propose some improvements in the measurement methods were used. For this purpose, it has been applied a theoretical measurement of the level of polycentricity within three regions of southern Chile: la Araucanía, los Ríos and los Lagos. The results suggest the existence of a lower middle polycentricity as a whole system and a low level of polycentric in each region.
\end{abstract}

Key words: Polycentrism, city systems, Chile.

Agradecemos al profesor de Economía de la Universidad Católica del Norte, Chile, Dr. Marcelo Lufin, por sus contribuciones en la propuesta y elaboración de Lmaxtd, así como también al Dr. N. Green (School of the Built Environment, University of Salford) y al Ing. Mg. David Poblete (Centro de Cambio Global UC), por sus sugerencias y aportes. Artículo recibido el 19 de mayo de 2011, aceptado el 20 de mayo de 2012 y corregido el 30 de junio de 2012.

2 Departamento de Geografía, Universidad de Concepción (Chile). E-mail : franmaturana@udec.cl

3 Instituto de Geografía, Pontificia Universidad Católica de Chile (Chile). E-mail : farenasv@uc.cl 
En el año 1999 la Unión Europea (UE) se planteó un proyecto de desarrollo territorial sustentable que buscaba balancear los territorios, buscando disminuir las disparidades al interior de esa macrorregión. Sin definirlo tácitamente, detrás de ese desafío estaba un concepto que ha tomado fuerza en el último tiempo, el policentrismo.

Es posible entender el policentrismo como una organización espacial basada en ciudades relativamente homogéneas, sin un dominio importante de alguna de ellas ni menos la existencia de una situación de macrocefalia, lo que, según la UE (1999), ayuda a consolidar una cohesión social y territorial. Para Gloersen (2005: 69) se trata de "una organización espacial de ciudades caracterizada por perfiles económicos urbanos diferentes, con una integración económica, institucional y de cooperación en políticas inter urbanas" 4 , o como destaca Davoudi (2007: 66), cuya definición se acerca al concepto de regiones urbanas policéntricas, donde "el policentrismo trata de una Región con tres o más ciudades que históricamente y políticamente han sido independiente, sin jerarquía y con una proximidad de ciudades que tienen un elevado grado de conexión y de complementariedades" ${ }^{5}$.

En esta publicación se comprende el policentrismo según las contribuciones planteadas por Spiekermann y Wegener (2004), en Green (2007: 2082) o cercana a la de Parr (2004). El primero señala que el policentrismo debe tener cuatro características principales:

- La existencia de una distribución de grandes y pequeñas ciudades (en nuestro caso sin grandes disparidades de talla).

- Una distribución rango-tamaño no linear.

- Una distribución rango-tamaño extendida no abrupta.

- La no dominación por parte de una gran ciudad.

A pesar de estas definiciones, el concepto de policentrismo no está todavía del todo estabilizado, básicamente porque ha sido enfocado desde distintas perspectivas, una que podríamos llamar europea (en la que se centra básicamente este artículo) y otra norteamericana; en efecto, según como sea comprendido, podríamos entender a la ciudad de Los Ángeles (EE.UU.) como una ciudad policéntrica (en vez de difusa), o también una organización como el Randsat en Holanda como policéntrica, donde cada centro urbano tiene una vocación funcional y no existen grandes diferencias en el tamaño de los centros urbanos.

El concepto también ha enfrentado críticas: según Rozenblat (2004: 140) "una red urbana densa y poco jerarquizada no es garantía ni de desarrollo económico, ni de equidad territorial" ${ }^{\prime 6}$. En este sentido, la búsqueda de una red equilibrada que conlleve una cohesión social, ha sido criticada (Baudelle y Peyrony, 2005; Baudelle y Montabone 2008; Meijers, 2008), ya que dentro de la política europea se confrontaría de manera contradictoria la búsqueda de competitividad territorial que reforzaría la idea de una polarización entre territorios.

Sin embargo, más allá de estas críticas, el concepto ha tomado cuerpo y la literatura científica se ha enfocado a medirlo básicamente en dos vertientes, las que podrían ser complementarias. La primera, de carácter morfológico y la segunda de tipo relacional o funcional (Carrière, 2005; Meijers, 2008; Yue et al., 2010). La primera está asociada a la distribución de las masas de población y las áreas urbanas sobre el territorio, y la segunda, más bien enfocada a las relaciones entre aglomeraciones, a los flujos funcionales que se desarrollan entre centros urbanos.

En cuanto a los enfoques morfológicos, es posible encontrar el programa ESPON 1.1.1 que utiliza una serie de indicadores o también a Riguelle et al. (2007), que utilizan el análisis de subcentros y densidad de empleo. Por otro lado, en los enfoques relacionales o funcionales encontramos contribuciones de modelos que se establecen para medir el grado de policentrismo a partir de los flujos, ejemplos de los cuales se encuentran en ESPON 1.1.1 (2005), POLYNET Action 1.1 (2005) y Green (2007).

\footnotetext{
6 Traducción de los autores. 
Para el caso chileno, las mediciones de policentrismo son escasas, destacándose dos publicaciones, una de Becerril-Padua (2000), para el caso de Santiago, y otra de Rojas et al. (2009), para el caso de Concepción, ambos centros urbanos con características de espacios metropolitanos, por lo que los autores enfocan el policentrismo desde un punto de vista más bien tipo norteamericano, considerando espacios metropolitanos de tipo difuso como elementos policéntricos. La perspectiva de este artículo está lejana a la consideración de un espacio metropolitano con sus satélites, como un sistema policéntrico, y más bien sigue la línea planteada por Spiekermann y Wegener o Davoudi.

Si los espacios metropolitanos en Chile han sido poco estudiados desde un enfoque policéntrico, en otros espacios la situación es aún más paupérrima. En el sur de Chile, los estudios a nivel regional en que se analizan varias ciudades de una o más regiones son escasos; de hecho, los estudios de interacción entre centros urbanos son casi inexistentes, salvo por el trabajo de Varela (2001), que analiza la interacción entre la ciudad de Valdivia y Temuco, o de Peña y Escalona (2009), o de Maturana y Cornejo (2010), donde se abordan estudios en centros urbanos de la Región de La Araucanía.

Hidalgo y Arenas (2009) ponen énfasis en la reproducción de mecanismos propios de los espacios urbanos metropolitanos, en ciudades medias regionales, incluyendo algunas del sur del país (Hidalgo et al., 2009). Otros estudios han sido planteados desde una óptica de los instrumentos territoriales, tales como los Planes Reguladores Intercomunales, los antiguos Planes Regionales de Desarrollo Urbano, los recientemente instalados Planes Regionales de Ordenamiento Territorial o los esfuerzos del Instituto Geográfico Militar, en el caso de la descripción de la estructura funcional de los sistemas urbanos regionales, en una serie de libros realizados para gran parte de las regiones del país, entre los años ochenta y noventa.

En este sentido, tres regiones del sur de Chile conforman un espacio de gran interés para el análisis de este tema, las regiones de La Araucanía, de Los Ríos y de Los Lagos
(Figura $\mathrm{N}^{\circ} 1$ ), con una posible estructura monocéntrica en su sistema urbano, dominado por las capitales regionales respectivas, las que serían polos concentradores de bienes y servicios. Para la Región de La Araucanía, esto último queda demostrado en el trabajo de Maturana y Cornejo (2010), en el que a partir de un análisis exploratorio de red se demuestra el rol determinante de la ciudad de Temuco en su espacio regional.

Lamentablemente no existen estudios que analicen el grado de cohesión en este sistema de tres regiones, a pesar del hecho que la Región de Los Ríos procede de una escisión de la Región de Los Lagos.

Dado lo anterior, es que se ha planteado en este trabajo medir el grado de policentrismo de manera exploratoria, a partir de los trabajos de Hall et al. (2006) y de Green (2007), los que plantean, según la teoría de grafos y métodos de análisis de redes, una metodología para medir el grado de policentrismo en un sistema de ciudades.

Así, los objetivos planteados en esta publicación están orientados a tres aspectos: primero, tratar de aportar y aplicar (de manera exploratoria) el método de medición de policentrismo utilizado por los autores mencionados en el párrafo precedente; segundo, comprender el grado de cohesión territorial que tiene el sistema de ciudades de las regiones de La Araucanía, de Los Ríos y de Los Lagos $y$, finalmente, demostrar la pertinencia y aplicabilidad de este método para un caso chileno.

En la Figura $\mathrm{N}^{\circ} 1$ es posible apreciar la localización del área de estudio, la que se despliega desde aproximadamente los 590 $\mathrm{km}$ al sur de Santiago y hasta los $1.100 \mathrm{~km}$ al sur de la misma.

\section{Metodología}

El método utilizado en este artículo está estructurado en tres etapas: la elección del número de ciudades, la definición de flujo utilizado y el cálculo de policentrismo, con propuesta de mejoras al método de Hall et al. (2006) y Green (2007). 


\section{Ciudades seleccionadas}

Camagni (2005) señala que al efectuar un análisis de sistemas de ciudades no es necesario tomar en cuenta centros urbanos de menor talla, ya que no tienen mayor injerencia en las dinámicas urbanas. Por lo anterior, se establecieron dos criterios con la finalidad de definir las ciudades a tomar en cuenta para el estudio: uno de tamaño demográfico del centro urbano (talla), y otro funcional. Respecto al primero, Maturana y Terra (2010) y Michel (1977) señalan que seleccionar ciudades y establecer criterios de corte a partir de sus tallas, corresponde a un análisis complejo. Para esta investigación se llevó a cabo lo siguiente:

Lo primero que se debe considerar es que dentro del área de estudio se localizan 40 ciudades (ver lista y población en el Cuadro $N^{\circ} 1$ ), según la definición de ciudad establecida por el Instituto Nacional de Estadísticas (INE) de Chile, que la define como una "entidad urbana que posee más de 5.000 habitantes" (INE, 2005: 11).

Para la población de los centros urbanos presentes en el sistema, se calcularon algunas medidas estadísticas con el fin de observar la distribución por talla de las ciudades y establecer un valor de corte.

Como se observa en el Cuadro $N^{\circ} 1, y$ como era esperable, no se está frente a una distribución normal; el promedio está bastante distanciado de la mediana. En efecto, la distribución de población de las ciudades del sistema se ve afectada por los cuatro grandes centros urbanos presentes en el sistema (Temuco, Puerto Montt, Valdivia y Osorno). Considerando el valor de Q3 (cuartil 3), el cual indica que aproximadamente el $75 \%$ de las ciudades tiene una talla menor a 22.000 habitantes, se piensa que el tamaño de corte debiera estar bajo esa cantidad de población, para hacer representativo el análisis. Tomando en cuenta el valor de cuartil 1 (Q1) y los valores de población, se decidió establecer como criterio de corte las ciudades sobre 9.000 habitantes, lo que corresponde aproximadamente al $76 \%$ de ciudades presentes en el sistema y aproximadamente al $97 \%$ de la población presente en todas las ciudades del sistema.
El segundo criterio para definir las ciudades es más bien funcional y consistió en establecer un buffer de $30 \mathrm{~km}$ alrededor de cada capital regional, definido a partir de la movilidad cotidiana que considera el programa ESPON y también a partir de los análisis de Peña y Escalona (2009), para el caso de Plan Regulador Intercomunal de Temuco. El buffer se estableció gracias al software (gratuito y libre) Quantum GIS. Según este criterio, solo se agregó la ciudad de Freire ya que los demás centros urbanos estaban incluidos según el criterio anterior. De este modo, treinta ciu-

\section{Figura $\mathrm{N}^{0} 1$}

Principales centros urbanos de las regiones de La Araucanía, Los Ríos y Los Lagos

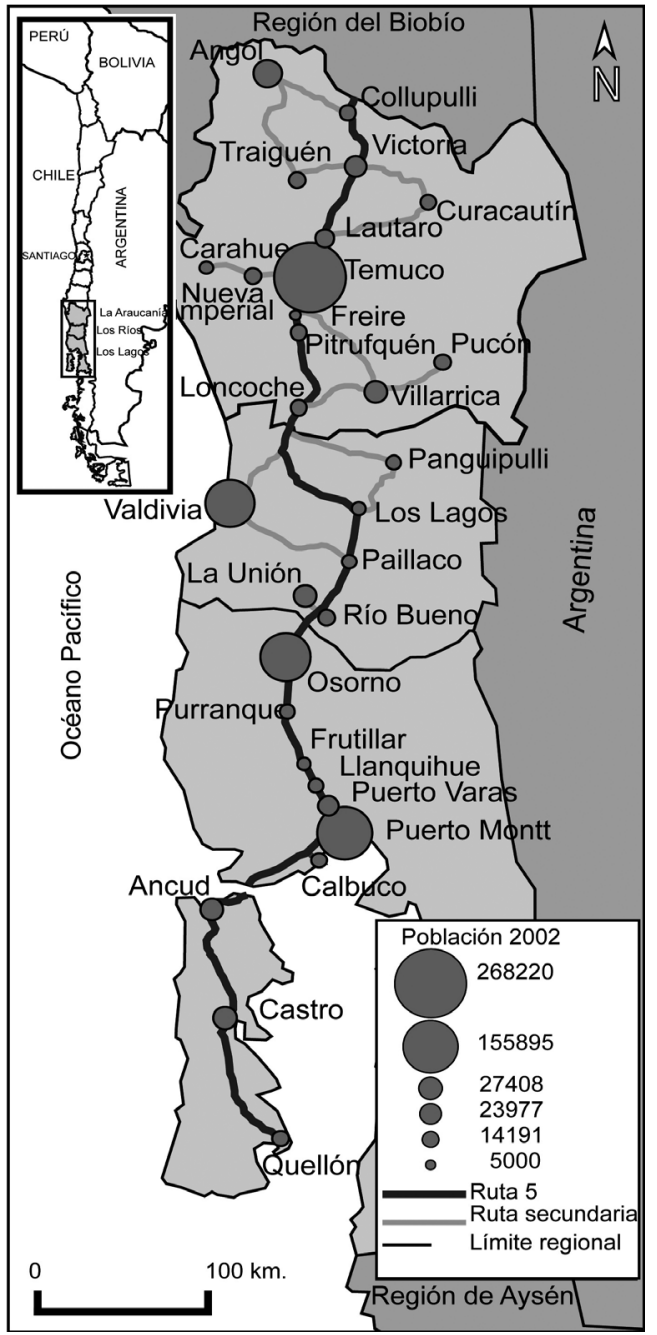

Fuente: Elaboración propia, a partir de INE, 2005. 


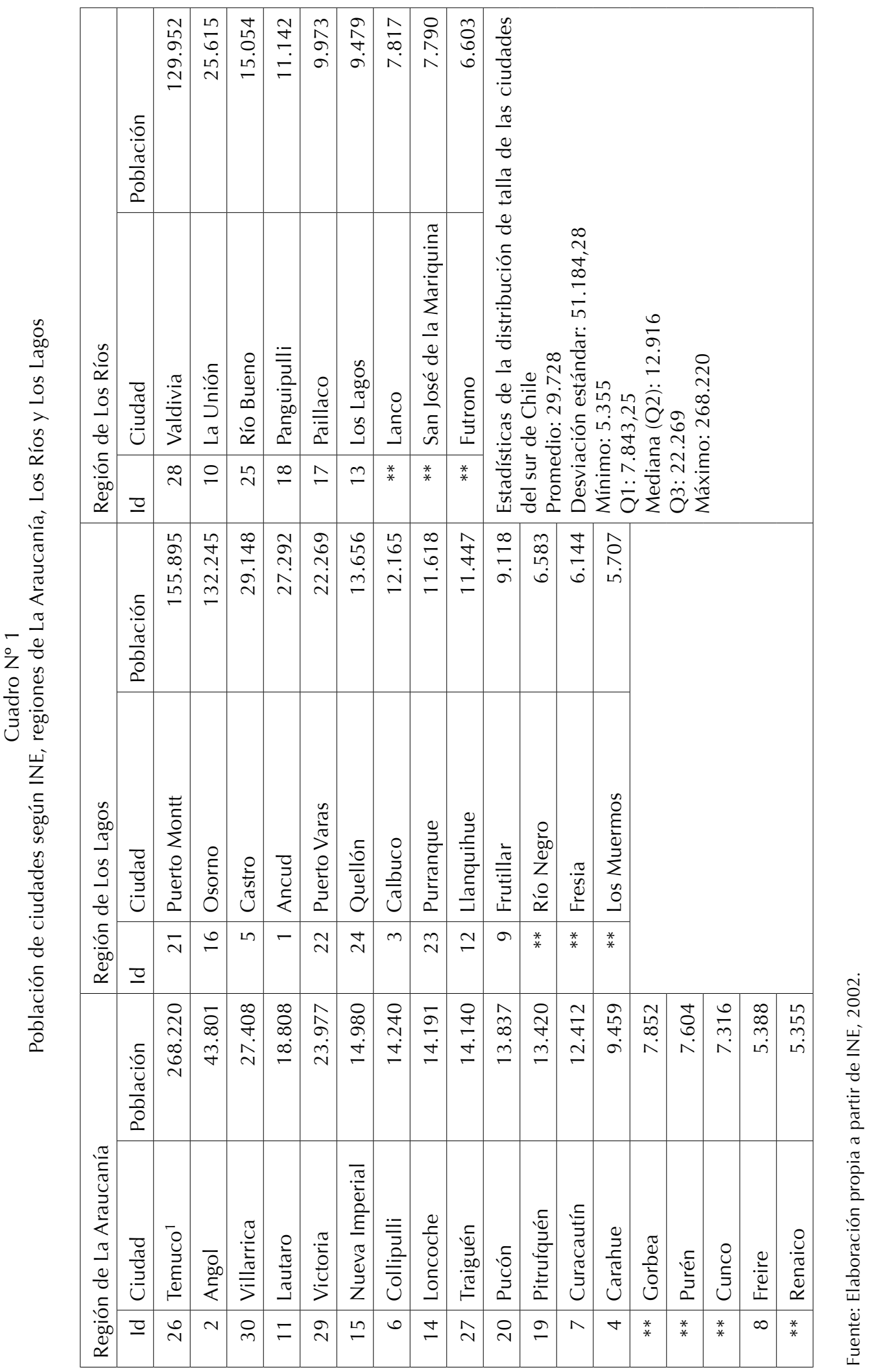


dades fueron seleccionadas para el análisis, las que están numeradas con un identificador en el Cuadro $\mathrm{N}^{\circ} 1$.

\section{Flujo utilizado}

Contar con datos de flujos entre centros urbanos es una tarea compleja en el caso de Chile, y cuando ellos existen, su acceso resulta dificultoso, especialmente cuando se trata de llamadas telefónicas o correos electrónicos, información a la que lamentablemente fue imposible acceder pese al contacto con las empresas del rubro, debido a que estos datos los consideran como confidenciales.

Respecto a otros datos de flujos, como por ejemplo de automóviles, las opciones disponibles eran censos viales del Ministerio de Transporte del Estado de Chile, pero que lamentablemente no son sistemáticos y no analizan de manera directa los flujos entre dos centros urbanos.

Dado lo señalado, para poder realizar la investigación se procedió a utilizar la pregunta $N^{\circ} 33$ del Censo de Población y Vivienda del año 2002 para las personas mayores de 15 años, que se refiere a la comuna donde se trabaja o estudia. Esta información ya fue utilizada para otro tipo de análisis por Maturana y Cornejo (2010) en la Región de La Araucanía, entregando resultados coherentes.

En este sentido, a pesar de las opiniones de Halbert et al. (2006) en cuanto a que para realizar este tipo de análisis (de interacción entre ciudades) es necesario utilizar flujos de empresas, numerosos estudios utilizan la movilidad laboral (con o sin estudio agregado) para este tipo de enfoques (Pumain \& Saint-Julien, 2001; Aguilera, 2005; Hall et al., 2006; Limtanakool et al., 2007; Green, 2007, 2008; y Hornis \& Van Eck, 2008).

Una vez determinadas las ciudades, se procedió a extraer la pregunta hecha a los habitantes censados para cada ciudad, en el censo 2002, gracias a las manzanas censales de cada una de ellas entregadas por el INE, las que fueron seleccionadas con el software REDATAM. Este paso tuvo como objetivo extraer la respuesta respecto a la comuna a la que se desplazan las personas por motivos de trabajo o estudio, incluyendo la totalidad de la población que se desplaza en cada ciudad. El problema que surgió fue saber a qué ciudad de la comuna llegaba el habitante. En efecto, se tuvo claro que se está tomando el $100 \%$ de la muestra en cuanto al origen de la persona (la ciudad), pero lamentablemente solo se podía saber a nivel comunal su llegada, por lo que se tomaron los siguientes supuestos:

Si el traslado es por motivos de estudios universitarios, las universidades en el área de estudio están localizadas solo en centros urbanos, siendo Temuco y Valdivia los más destacables (Consejo de Rectores, 2009). En cuanto a la enseñanza media, datos del Ministerio de Educación (2009) sugieren que también la mayoría de los establecimientos se concentra en los centros poblados. Además, en el caso que la persona indicase que se desplaza a una comuna por motivos de estudio medios, era ilógico pensar que un estudiante iría desde la ciudad hacia una escuela rural, con todas las dificultades en transporte, accesibilidad (especialmente en invierno) y donde la calidad de la educación probablemente sea inferior. Respecto a la movilidad laboral, se cree que es una variable que no es posible manejar $y$, por ende, una debilidad en el dato. La movilidad laboral desde una ciudad hacia una zona rural podría estar dada por la estacionalidad de algunas actividades económicas, pero se tomó el supuesto que la mayoría de los desplazamientos por este motivo son a un centro urbano, en este caso particular, la ciudad presente en la comuna. Se debe destacar que en el área de estudio cada comuna posee solamente una ciudad, según la definición dada por el INE.

Como último punto, es necesario tener en consideración que con este dato se está tomando el cien por ciento de la población ya que sería muy difícil y caro obtener una muestra correcta por medio de una encuesta.

De esta forma, y a partir de estos supuestos, se pudo obtener un dato de flujo entre un centro urbano y una comuna, asumiendo que en esta última el destino del flujo será la ciudad existente en ella.

Así se construyó la matriz de datos del Cuadro $\mathrm{N}^{\circ} 2$, cuyo identificador se puede ver en el Cuadro $\mathrm{N}^{\circ} 1$. 


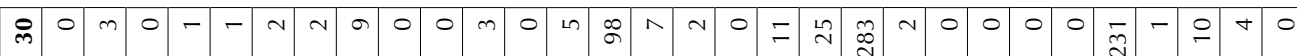

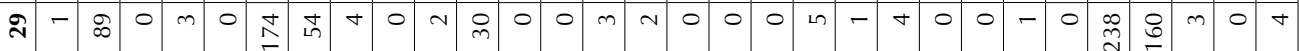

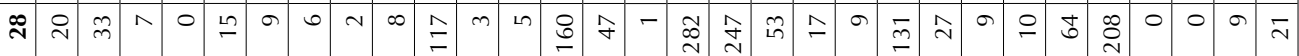
ล 0 이

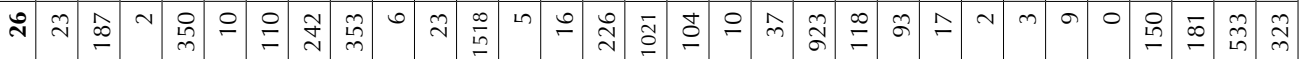

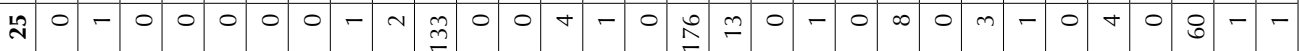

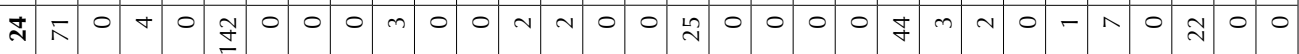

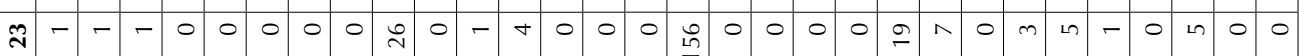

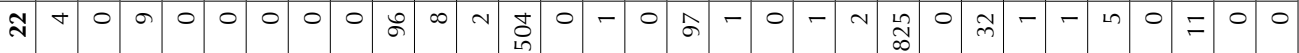

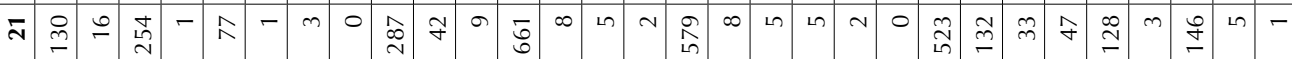
今

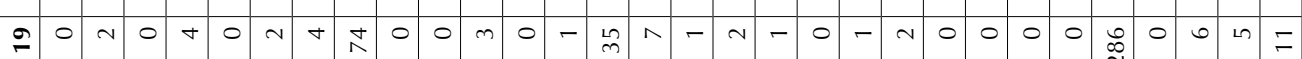

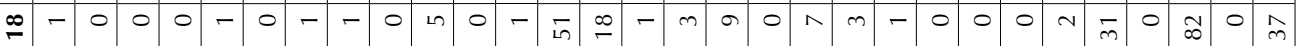
N ○

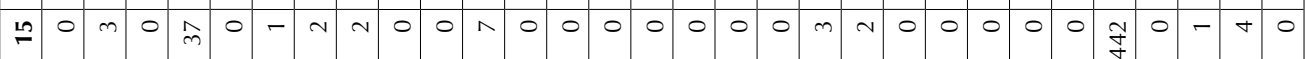
I m

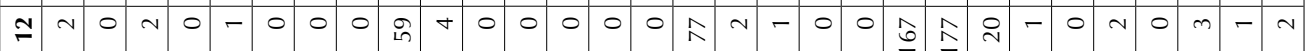

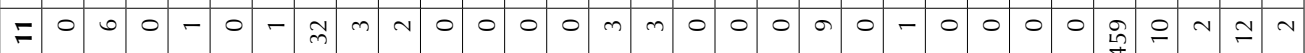
o -0 -

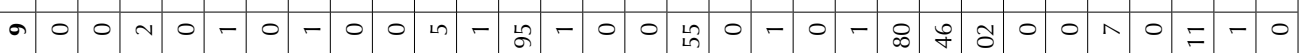

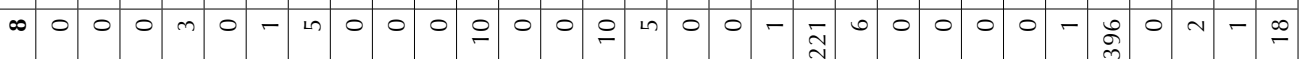

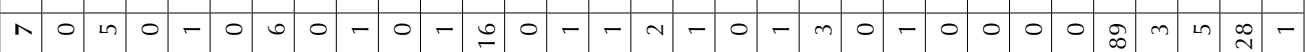

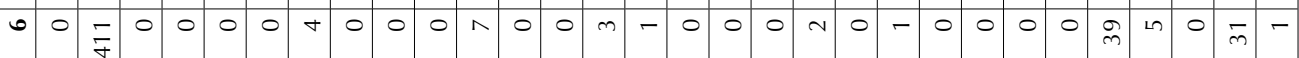
n

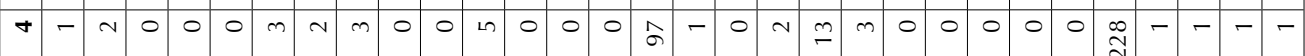

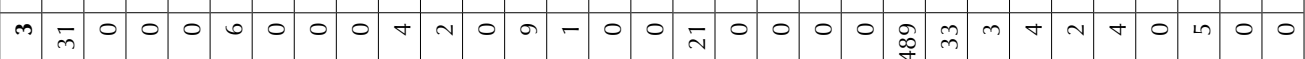

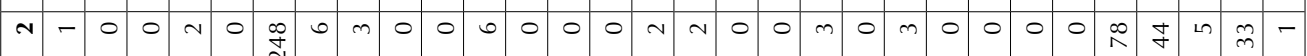
- 0 o

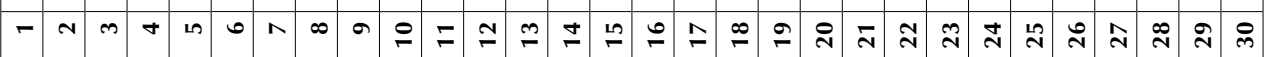


Cálculo de policentrismo y propuesta de mejoras al método de Hall, Pan y Green (2006) y Green (2007)

Para medir el grado de policentrismo de un sistema de ciudades desde un enfoque funcional o relacional, se quiso modificar y contribuir a la propuesta realizada por Hall, Pain y Green (2006) y Green (2007), las cuales están basadas en la teoría de grafos y métodos de análisis de redes.

En este sentido es necesario comprender lo siguiente:

a) Densidad de un grafo, la que se puede definir como la proporción de líneas posibles que son realmente presentadas en el grafo, o también como la relación entre el número de líneas presentes y el máximo posible (Wasserman y Faust, 1994).

Así la densidad del grafo queda representada según la ecuación 1 (Wasserman \& Faust, 1994):

$$
\Delta=\frac{\mathrm{L}}{\operatorname{Lmax}}
$$

\footnotetext{
$\Delta=$ Densidad del grafo.

$L=$ Suma de todos los flujos reales entre los nodos.

Lmax = Cantidad máxima de flujos teóricos posibles entre los nodos.
}

Como se puede observar, el problema de la definición de densidad de un grafo radica en delimitar la cantidad máxima de flujos posibles (Lmax). Como es sabido, la red sobre la que trata este artículo es una red orientada (ya que un flujo puede ir de una ciudad $A$ hacia una $B$, aunque no necesariamente el centro urbano $B$ enviará flujos hacia A), y valorada, donde el valor del flujo es una magnitud, que en este caso corresponde al número de personas con desplazamientos cotidianos por motivos de trabajo o estudio.

El punto radica en que no se puede olvidar que existe población residente en la ciudad que potencialmente se podría desplazar; entonces ¿cómo calcular la densidad de la red? ¿Debemos agregar la población que trabaja o estudia en una misma ciudad en nuestra red? ¿Qué es lo que sucede si solo se cuenta con flujos de correos electrónicos o llamadas telefónicas, por ejemplo? ¿Será sencillo delimitar el máximo de flujos posibles? La respuesta a estas interrogantes es que la complejidad y cálculo de Lmax será en función del flujo a utilizar. En efecto, Green (2007) enuncia este problema, pero la paradoja que se encuentra en la propuesta de este autor es que el Lmax que presenta puede ser poco representativo de la población que trabaja; además, en la medición de policentrismo el Lmax es una variable muy sensible.

La complejidad en el cálculo de Lmax en la ecuación 1 radica en que, por ejemplo, si se posee flujos de correos electrónicos no sería fácil establecer un máximo de flujos posibles y requeriría estimaciones complejas para ser establecido, del mismo modo en el caso de las Ilamadas telefónicas.

Para el caso de la movilidad cotidiana por motivo de trabajo o estudio, a pesar que la estimación de Lmax es compleja, sí se puede saber la cantidad máxima de flujos posibles en cada nodo, ya que será la población que trabaja o estudia (mayor de 15 años) en la ciudad misma, más los flujos que realiza a otras ciudades.

Por tanto, el primer objetivo fue calcular el Lmax de la manera más representativa posible, tratando de identificar la población máxima potencial que realizará un desplazamiento por motivo de trabajo o estudio.

Green (2007) calcula un Lmax a partir de la población activa. Este autor suma todas las poblaciones activas de los centros urbanos y resta el menor valor de las ciudades presentes.

Este método para calcular el Lmax no estaría tomando la tendencia observada en el sistema de ciudades, y además, para el caso de los datos obtenidos del INE, resulta dificultoso utilizar este método de cálculo de Lmax, debido a que se incorporó población por desplazamiento de estudio y porque la definición de población puede diferir, por lo que para calcular un nuevo Lmax se propone lo siguiente: 


$$
\Delta=\frac{\mathrm{L}}{\operatorname{Lmaxtd}}
$$

Donde:

$$
\Delta=\text { Densidad del grafo. }
$$

$L=$ Suma de todos los flujos reales entre los nodos.

Lmaxtd= Cantidad máxima de flujos teóricos realizables entre nodos a partir de la tendencia observada.

Para calcular Lmaxtd es necesario calcular el máximo de la normalización de los flujos:

$$
M f n=\operatorname{Max}\left(\frac{f v_{i}}{\sum P m q_{i}}\right)
$$

Donde:

$M f n=$ Es el máximo de los flujos normalizado para todas las ciudades, es decir, es el porcentaje máximo de movimiento de flujos de movilidad cotidiana en todas las ciudades del sistema.

$f v=$ Flujo real para cada ciudad $\mathrm{i}$

$\mathrm{Pmq}_{i}=$ Suma total de flujos reales de cada ciudad con la población total que trabaja o estudia en cada ciudad i.

Seguido, se tuvo que calcular el mínimo en términos de personas que se desplazan:

$$
M p=\operatorname{Min}\left(M f n \times P m q_{i}\right)
$$

Donde:

$M p=$ Más pequeño número de flujos normalizados en términos de número de personas que se desplazan.

Para finalmente,

$$
L \operatorname{maxtd}=\operatorname{Max}\left(\left(M f n \times P m q_{i}\right)-M p\right)
$$

Se debe hacer notar que fueron tomados los bucles o loops ${ }^{7}$ de trabajo o estudio de cada ciudad para estimar el Lmaxtd.

\footnotetext{
7 Es el flujo que conecta con el mismo nodo, en este caso, la población de la ciudad que trabaja o estudia en la misma ciudad.
}

Así, el resultado de la ecuación 5 es un valor máximo calculado y limitado a la tendencia actual observada en el sistema de ciudades y no sobre una población teórica basada únicamente en la población que reside en el centro.

A pesar que se comprende que el cálculo presentado de este Lmaxtd puede ser criticado y mejorado, las discusiones sostenidas con N. Green apuntaban a mejorar estos aspectos. Se cree que el enfoque enunciado es una contribución en esta dirección, a pesar que sería necesario profundizarlo.

Para continuar con la medición, se retoma la propuesta de Hall, Pain \& Green (2006) y Green (2007), donde es necesario calcular la Special Functional Polycentricity.

La Special Functional Polycentricity para el grado interior y exterior para cada nodo (ciudad) ${ }^{8}$ :

$$
\operatorname{Psf}(\mathrm{N})=\left(1-\frac{\sigma}{\sigma \max }\right) \times \Delta
$$

Donde:

Psf = Es la función policéntrica especial para una función $f$ contenida en una red $N$ : $\sigma$ es la desviación estándar del grado del nodo; $\sigma$ max es la desviación estándar del grado del nodo en una red de tipo $2\left(n_{1}, n_{2}\right)$ provienen de $N$ donde $d n_{1}=0$ y $d n_{2}$ = al valor del nodo con el más alto valor en $N$ y $\Delta$ es la densidad del grafo (Green, 2007: 2085).

La fórmula de Special Functional Polycentricity es sostenida según tres criterios:

a) El policentrismo es definido como una función de red teórica y no toma en cuenta las distancias físicas entre los nodos, por tanto es evolutiva.

b) La definición comprende la densidad de la red, lo que significa que el nivel de interacción entre los lugares, en términos de una función particular, es considerado y

8 Grado interior es el número de entradas hacia el nodo y grado exterior es el número de salidas desde el nodo. 
tiene entonces una incidencia en la medida en la que una red de una ciudad puede ser considerada como un sistema único.

c) El policentrismo fue definido en términos funcionales y puede entonces ser utilizado para describir policentrismo en una variedad de funciones en una misma zona geográfica. Es decir, una variedad de relaciones funcionales pueden ser medidas y comparadas por un conjunto de nodos. Se trata de estimar niveles de policentrismo a partir de flujos telefónicos, correos electrónicos, movilidad de personas, etc., en un mismo sistema y área geográfica y con un mismo método, de manera de poder comprender una estructura de forma más profunda.

Finalmente, es necesario calcular la ecuación general funcional de policentrismo de una red (Fuente: Green 2007: 2086):

$$
\mathrm{P}_{G F}\left(\mathrm{~N}_{1}, N_{2} \ldots \mathrm{N}_{\mathrm{n}}\right)=\frac{\sum_{n=1}^{\mathrm{n}} \mathrm{P}_{G F}\left(\mathrm{~N}_{1}, N_{2} \ldots \mathrm{N}_{\mathrm{n}}\right)}{\mathrm{n}}
$$

Donde: $\mathrm{P}_{G F}\left(\mathrm{~N}_{1}, N_{2} \ldots \mathrm{N}_{\mathrm{n}}\right) \begin{aligned} & \text { es la ecuación general funcional policentri- } \\ & \text { ca para una red funcional. }\end{aligned}$

$\mathrm{N}_{1}, N_{2} \ldots \mathrm{N}_{\mathrm{n}}, P_{S F}\left(\mathrm{~N}_{1}, N_{2} \ldots \mathrm{N}_{\mathrm{n}}\right) \begin{aligned} & \text { son los valores para la } \\ & \text { policentricidad fun- }\end{aligned}$ cional para las redes.

$\mathrm{N}_{1}, N_{2} \ldots \mathrm{N}_{\mathrm{n}} y \mathrm{n}$ es el número de redes.

Para Green (2007) esta fórmula no estaría completa y sería necesario agregar un modificador de complementariedad cuando existen más de tres redes. Para este artículo se constató que era irrelevante dado los resultados obtenidos y también según los valores obtenidos en el programa POLYNET.

Finalmente, los valores de policentrismo estarán comprendidos entre 0 y 1 , y en cuanto más cercano a 1 habrá más policentrismo. Pero a pesar de estos valores y, como fue observado en el programa POLYNET para una parte de territorio europeo, los valores obtenidos fueron bastante más bajos: entre 0,020 y 0,250 (sin modificador de complementariedad).
Los resultados con valores de bajo policentrismo sería posible explicarlos por el Lmax utilizado. Como se verá más adelante, con el Lmaxtd los valores estarían dentro de lo esperado; en efecto, si el sistema de ciudades tiene una suma total de flujos reales aproximados al 15\% del total del Lmaxtd calculado, habrá un bajo policentrismo cercano a 0,1 (calculado a partir de una red de más de 10 ciudades), lo cual se traduce en una red más bien monocéntrica, donde la dominancia de una ciudad es evidente. Si, por ejemplo, los flujos reales son aproximadamente un $50 \%$ del total de Lmaxtd, el grado de policentrismo será próximo a 0,3. Esto significa que para la existencia de policentrismo al menos la suma de flujos reales debería ser superior al valor obtenido de Lmaxtd (con un valor igual se estaría cercano a un 0,57$)$. Surgen inmediatamente dos simples dudas: ¿es posible obtener este valor 0,57 o más?, ¿con un valor de 0,57 o más se puede observar cierta tendencia policéntrica?

Teóricamente, para el modelo que está propuesto si se quisiera elevar el grado de policentrismo de una red (partiendo de la hipótesis que es bajo), sería necesario que los habitantes que trabajan o estudian en la ciudad con más flujos del sistema (que generalmente recibe más flujos de los que envía), por diferentes razones (una política territorial que tuvo impacto en la localización de una industria en otra ciudad, por ejemplo), se desplacen hacia otra ciudad (de menos flujos en el sistema). Por tanto, el Lsum tendrá un aumento en su magnitud superior al aumento de Lmaxtd (que también tendrá un aumento pero menor) y, por ende, el valor de la densidad del grafo (ecuación 2) también se eleva tal como el grado de policentrismo. Por el contrario, si en el mismo sistema son las ciudades con menor magnitud de flujo las que aumentan los desplazamientos, el grado de policentrismo se mantiene prácticamente igual.

No se trata, estrictamente hablando, de que los habitantes de centros urbanos de mayor flujo (que podrían tener mayor cantidad de población) deban desplazarse a otros centros de menor cantidad o con menor flujo para así generar un mayor policentrismo. Perfectamente se podría dar una migración (no un desplazamiento o movilidad cotidiana), 
desde centros de mayores flujos (asociados a los de mayor talla), hacia los centros de menor talla, y así estos últimos aumentarían en población, el gran centro disminuiría su talla y se podría tender a equilibrar los flujos (según los bucles), obviamente todo dentro de un contexto de políticas territoriales, y salvo un evento externo no considerado (terremoto, tsunami, guerra, etc.).

Las conclusiones que se pueden obtener de lo expuesto y de los elementos desarrollados es que existirá una correcta interpretación del método, dado lo que acá se ha considerado como policentrismo y sería necesario tener en cuenta los siguientes elementos en dicho cálculo:

a) Si la desviación estándar aumenta, disminuye el policentrismo. En efecto, las desviaciones estándar ayudan a identificar la homogeneidad de los flujos en las relaciones entre ciudades (ellas toman la concentración o su opuesto en las relaciones de la red). Entonces mientras mayor homogeneidad habrá mayor policentrismo y mientras más relaciones (flujos entre ciudades) aumenta el policentrismo.

b) Si la densidad del grafo aumenta, aumenta el policentrismo.

c) La densidad del grafo mediría la amplitud en relación con los vínculos reales y con los máximos teóricos posible; ella sería subordinada al cálculo de Lmaxtd que será una variable sensible en el método.

La propuesta de Hall et al. (2006) y Green (2007) y que se ha planteado mejorar partir del cálculo de Lmaxtd, se sostiene sobre la idea de un policentrismo relacional en un sistema de ciudades, que en un óptimo conlleva la idea de que hay centros urbanos que tienen la misma capacidad de enviar como de recibir información, donde no hay una notoria concentración de trabajo y educación, donde existe una cooperación entre centros urbanos; algo cercano al concepto francés de réseaux de villes (redes de ciudades).

Cuadro $\mathrm{N}^{\circ} 3$

Sumas de flujos totales más bucles por ciudad

\begin{tabular}{|l|r|r|r|l|r|r|r|}
\hline Ciudad & \multicolumn{1}{|l|}{$\begin{array}{l}\text { Suma flujos } \\
\text { flux }\end{array}$} & Bucles & \multicolumn{1}{l}{$\begin{array}{l}\text { Suma } \\
\text { total }\end{array}$} & Ciudad & $\begin{array}{l}\text { Suma flujos } \\
\text { flux }\end{array}$ & Bucles & Suma total \\
\hline Ancud & 437 & 10.069 & 10.506 & Osorno & 1.911 & 46.327 & 48.238 \\
\hline Angol & 782 & 12.482 & 13.264 & Paillaco & 388 & 2.917 & 3.305 \\
\hline Calbuco & 312 & 4.525 & 4.837 & Panguipulli & 134 & 3.720 & 3.854 \\
\hline Carahue & 407 & 2.764 & 3.171 & Pitrufquén & 1.274 & 2.953 & 4.227 \\
\hline Castro & 306 & 10.824 & 11.130 & Pucón & 441 & 4.144 & 4.585 \\
\hline Collipulli & 584 & 3.182 & 3.766 & Puerto Montt & 2.281 & 62.202 & 64.483 \\
\hline Curacautín & 384 & 2.993 & 3.377 & Puerto Varas & 1.893 & 6.626 & 8.519 \\
\hline Freire & 566 & 1.313 & 1.879 & Purranque & 661 & 3.013 & 3.674 \\
\hline Frutillar & 547 & 2.832 & 3.379 & Quellón & 139 & 5.910 & 6.049 \\
\hline La Unión & 870 & 7.168 & 8.038 & Río Bueno & 915 & 3.901 & 4.816 \\
\hline Lautaro & 1.645 & 4.445 & 6.090 & Temuco & 3.312 & 97.984 & 101.296 \\
\hline Llanquihue & 1.317 & 2.839 & 4.156 & Traiguén & 379 & 3.741 & 4.120 \\
\hline Loncoche & 502 & 3.542 & 4.044 & Valdivia & 1.232 & 51.896 & 53.128 \\
\hline Los Lagos & 311 & 2.672 & 2.983 & Victoria & 736 & 6.975 & 7.711 \\
\hline Nueva Imperial & 1.160 & 4.100 & 5.260 & Villarrica & 894 & 8.896 & 9.790 \\
\hline
\end{tabular}

Fuente: Elaboración propia a partir de INE, 2002. 
Por lo anterior, es que se cree que este método por sí solo no tendría la capacidad de identificar policentrismo y sería necesario complementarlo con otras variables o indicadores para poder justificar los resultados que se obtengan en la realidad. Es por esto que posiblemente sería imperativo un análisis de policentrismo desde el punto de vista morfológico, por ejemplo.

\section{Policentrismo de las ciudades del sur de Chile}

En el Cuadro $\mathrm{N}^{\circ} 3$ se puede observar los valores utilizados para el cálculo de Lmaxtd. A partir de la matriz de datos obtenida y la metodología planteada, en el Cuadro $\mathrm{N}^{\circ} 4$ se observan los resultados obtenidos de la medición del grado de policentrismo. Como se aprecia, el grado de policentrismo obtenido para todo el sistema fue de 0,49 , lo que sugiere la existencia de un medio-bajo policentrismo, el que se considera esperado ya que en este sistema se encuentran cuatro centros urbanos de gran tamaño: Temuco, Valdivia, Osorno y Puerto Montt y una elevada disparidad respecto del resto (como se aprecia en el cuadro $\mathrm{N}^{\circ} 1$, Valdivia, ciudad de menor población entre los cuatro centros nombrados, posee una población tres veces superior a la ciudad de Angol). A pesar de que no existe una macrocefalia, las relaciones monocéntricas en el sistema de ciudades de La Araucanía (demostrado por Maturana y Cornejo, 2010), y posiblemente en menor medida en las regiones de Los Ríos y Los Lagos, quedan reflejadas al obtener un valor de policentrismo medio-bajo.

\section{Cuadro $\mathrm{N}^{\circ} 4$}

Grado de policentrismo en el sistema de ciudades de las regiones de La Araucanía, Los Ríos y Los Lagos

\begin{tabular}{|r|l|r|}
\hline Etapas & Características & \multicolumn{1}{l|}{ Valor } \\
\hline 1 & Lsum & 26.720 \\
\hline 2 & Lmaxtd & 31.504 \\
\hline 3 & Densidad del grafo & 0,848 \\
\hline 4 & Desviación estándar grado interior & $1.301,109$ \\
\hline 5 & Desviación estándar grado exterior & 708,282 \\
\hline 6 & Psf grado interior & 0,513 \\
\hline 7 & Psf grado exterior & 0,485 \\
\hline 8 & Pgf & 0,499 \\
\hline
\end{tabular}

Fuente: Elaboración propia.
El cálculo de policentrismo efectuado obliga a reflexionar respecto de si realmente los resultados son concluyentes, por lo que se realizó de manera teórica el ejercicio de calcular el grado de policentrismo en una red "imaginaria", donde fueron suprimidos los cuatro grandes centros urbanos de la red, esto es, Temuco, Puerto Montt, Valdivia y Osorno.

Como se ve en el Cuadro $\mathrm{N}^{\circ} 5$, los valores arrojan un elevado policentrismo $(0,82)$, esto en concordancia a un sistema de ciudades donde no existen macrocefalias, por lo que los flujos que establecen los centros urbanos, en este "sistema imaginario", son equilibrados, lo que también iría en concordancia con el tamaño de las poblaciones y con una distribución que es más bien normal. A pesar de esto, se deben tomar con mesura los resultados y plantearse la siguiente interrogante: ¿realmente este resultado es reflejo de un elevado grado de policentrismo? Tanto desde el punto de vista de una red urbana equilibrada, como desde el punto de vista de flujos, la respuesta es positiva, aunque no se podría afirmar tajantemente que exista un grado de cooperación de ciudades o de relaciones horizontales no jerárquicas, como las planteadas por Dematteis (1997) o Camagni (2005). Más aún, suprimiendo los cuatro grandes centros del sistema, los servicios de salud, educación, etc., no estarían del todo cubiertos, por tanto el bienestar de la población posiblemente sería inferior a la situación "real". Por ende, parece necesario complementar este tipo de análisis con otras variables, que nos indiquen grados de asociatividad entre las ciudades en materia de políticas territoriales formales y datos de infraestructura, entre otros.

Cuadro $\mathrm{N}^{\circ} 5$

Grado de policentrismo red imaginaria

\begin{tabular}{|r|l|r|}
\hline Etapas & Características & \multicolumn{1}{|l|}{ Valor } \\
\hline 1 & Lsum & 6.108 \\
\hline 2 & Lmaxtd & 3.607 \\
\hline 3 & Densidad del grafo & 1,693 \\
\hline 4 & Desviación estándar grado interior & 170,421 \\
\hline 5 & Desviación estándar grado exterior & 158,976 \\
\hline 6 & Psf grado interior & 0,822 \\
\hline 7 & Psf grado exterior & 0,826 \\
\hline 8 & Pgf & 0,824 \\
\hline
\end{tabular}

Fuente: Elaboración propia. 
En este sentido, y a manera de complementar los resultados, es posible ver en la Figura $\mathrm{N}^{\circ} 2$ los flujos que se desarrollan entre las ciudades y las comunas, y constatar que las ciudades de mayor tamaño son centros receptores de flujos y las ciudades aledañas a estas, son "expulsoras". En el caso de la Región de La Araucanía, resulta muy claro para las ciudades de Lautaro, Nueva Imperial y Pitrufquén, centros expulsores de población, que posiblemente cumplen la función de ciudad dormitorio por su proximidad geográfica a la capital regional (no más de $40 \mathrm{~km}$ ).

En la Región de Los Ríos, los casos de Río Bueno y La Unión Ilaman profundamente la atención, ya que al observar la figura se constata como ambos centros urbanos son expulsores de población, pero si se observa con detenimiento la matriz de datos en el Cuadro $N^{\circ} 2$ se puede observar como la ciudad de Río Bueno está totalmente supeditada en términos de flujos a la ciudad de La Unión, y a su vez, ambos centros urbanos son totalmente dependientes de la ciudad de Osorno (la que se encuentra en la Región de Los Lagos) y no de su capital regional (Valdivia), como sería esperable. Este hecho remarca la importancia de este tipo de análisis en la planificación territorial, y cabe interrogarse respecto de si los instrumentos territoriales aplicados en las regiones de Los Ríos o Los Lagos consideran este tipo de "dependencia" entre centros, la influencia en los habitantes que residen en estas ciudades y las implicancias en términos de transporte y servicios, entre otras.

Otro aspecto a destacar, dada la Figura $\mathrm{N}^{\circ}$ 2 y el Cuadro $\mathrm{N}^{\circ} 2$, es que queda en evidencia el control al que está sujeto el sistema de ciudades del sur de Chile, por parte de algunos nodos (capitales regionales), así como la dependencia y vinculación de ciertos centros (ciudades dormitorio) con los más poderosos. Otro aspecto de interés es la existencia de otro conjunto de centros urbanos, cuya trayectoria no es el del todo clara, ya que al analizar las interacciones entre los centros urbanos de diferentes regiones en la matriz expuesta en el Cuadro $\mathrm{N}^{\circ} 2$, queda de manifiesto la ausencia de conexiones poderosas entre centros urbanos de diferentes regiones (salvo

Figura $\mathrm{N}^{\circ} 2$

Movilidad cotidiana en el sur de Chile

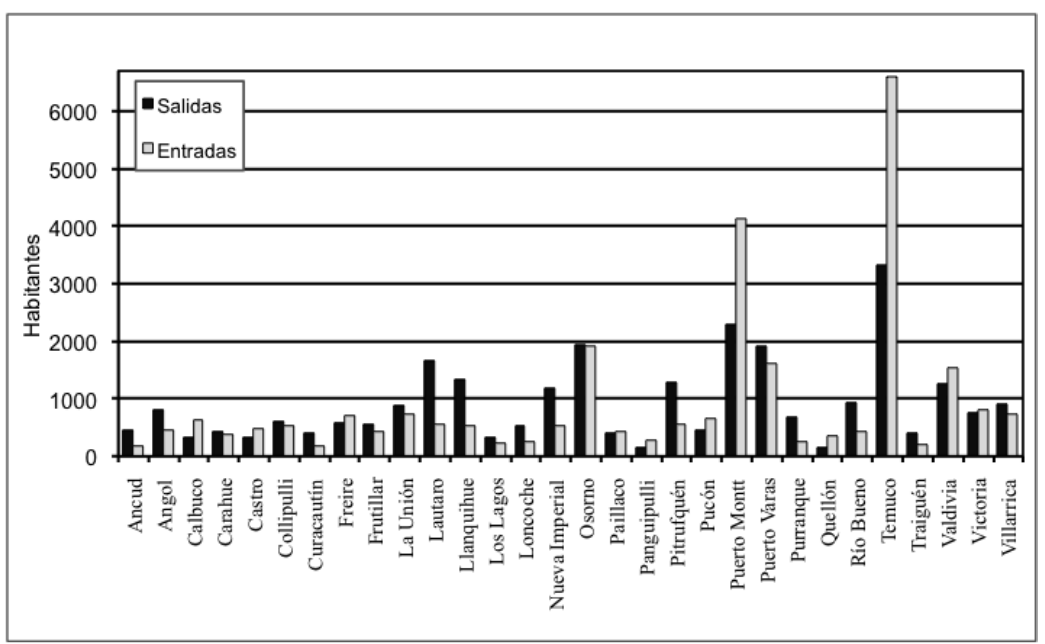

Fuente: Elaboración propia a partir de INE, 2002. 
las ya nombradas), por lo que se concluye que la mayoría de los vínculos, así como los más poderosos, se realizan al interior del espacio regional.

Si bien esta situación es esperable, ella deja también entrever como ha existido un desarrollo orientado al reforzamiento de las estructuras de vínculos al interior de espacios cerrados (región), donde la configuración de las relaciones entre los centros urbanos del sistema, estaría bajo la óptica de espacios de competencia, en que la atracción de recursos y personas vía distintas políticas, determinará su grado de inserción en la red y su posición en cuanto a su subordinación en la estructura territorial, generando relaciones de dominancia, concentración del poder, control de la información y existencia de nodos aislados.

Otro elemento que puede ayudar a corroborar el medio-bajo policentrismo presente y la disparidades espaciales que se han resaltado, es la Figura $\mathrm{N}^{\circ} 3$, donde se aprecia la curva rango-tamaño en relación a la curva "óptima" teórica. Es destacable en dicha figura como en 1940 la jerarquía urbana del sistema de ciudades del sur de Chile es desequilibrada, aunque las primeras ciudades no dominan de sobremanera al resto del sistema. En efecto, aparece equilibrada, más próxima a la curva "ideal". La dominancia de Temuco respecto al resto del sistema no parece excesivamente importante, como en el caso de Santiago a nivel nacional o París en Francia. A pesar de esto, resulta evidente que la curva del año 2002 aparece menos "ideal" y más desequilibrada, lo que estaría en concordancia con los resultados obtenidos de policentrismo de una jerarquía urbana medianamente desequilibrada.

Estos hechos quedan demostrados si se analiza con detenimiento los valores de la Figura $N^{\circ}$ 3. En el año 1940 la ciudad de Temuco era 1,2 veces más grande que la segunda, 1,6 más que la tercera, 1,9 veces superior a Puerto Montt y 3,3 veces a la ciudad de Angol. Al año 2002, la situación parece acentuada drásticamente, Temuco es 1,7 veces superior a Puerto Montt como segunda ciudad en la jerarquía y posteriormente 2,1 veces la tercera; 2,1 veces la cuarta y 6,1 la quinta, es decir, Angol.
Si se reflexiona a partir de estos simples cálculos (y de aquellos incluidos en el cuadro en anexo), es posible corroborar lo que plantea Dauphiné (2003) y Pumain \& Saint-Julien (2001), en cuanto a que los sistemas de ciudades son relativamente estables en el tiempo, respecto a la jerarquía que desarrollan (en términos de la cantidad de población). Lo dramático, en este caso, no es que el sistema siga relativamente estable (lo cual es totalmente esperado en términos de su jerarquía), sino que existe una acentuación en la disparidad de los centros urbanos al interior del sistema (o al interior de cada espacio regional), lo que se traduce en una ampliación de las desigualdades territoriales y, por ende, en nefastas consecuencias para la población residente, la cual posiblemente no solo se ve afectada por una dependencia en términos de servicios y otros, sino que también en términos de capacidad de decisión y oportunidades. El marco de acción de los habitantes de centros pequeños queda totalmente supeditado a una "ciudad central", la que al concentrar instituciones y recursos, puede "manejar" y "controlar" a otros centros de la red. Al tratarse de sistemas altamente estables en el tiempo, la trayectoria de estas de ciudades del sur de Chile al interior de cada espacio regional, podría estar reforzando de manera importante los desequilibrios constatados.

Por lo anterior, resulta interesante interrogarse acerca de la efectividad de los instrumentos territoriales aplicados, los que si bien han sido desarrollados con énfasis en los últimos años, no parecen tener un impacto en la estructura jerarquizada y más bien se disipan en la continua trayectoria hacia a una ruptura en la cohesión de las ciudades.

Si se considera, por ejemplo, un rápido análisis del periodo 1992 y 2002, en el que se comenzaron a aplicar instrumentos territoriales, para el año 1992 Temuco era 5,44 veces mayor que la ciudad de Angol, lo que si se contrasta con el año 2002, muestra claramente un aumento sustancial de la disparidad de 0,69 (ver Cuadro $N^{\circ}$ 6), al considerar que el tamaño de Temuco pasó a ser 6,1 veces superior al de Angol para el año 2002. Por lo tanto, es posible considerar que en ese periodo $y$, a pesar de las políticas territoriales, no han existido cambios sustanciales en 
el aumento de la cohesión entre los centros urbanos, sino más bien un aumento de las disparidades.

Si bien se entiende que el cambio de ciertas trayectorias urbanas en un periodo de 10 años puede ser limitado, para el caso de la Región de La Araucanía parece quedar de manifiesto al observar en el Cuadro $\mathrm{N}^{\circ} 6$ los diferentes saldos obtenidos por la supremacía demográfica de Temuco sobre Angol, en seis períodos de tiempo. Destacan el período 1960-1970 con un $0,88(4,75-3,87)$ y el más reciente 1992-2002, con 0,69 $(6,12-5,44)$. En este sentido, será muy interesante contar con los datos del censo de 2012, para observar si el proceso continua agudizándose.

Un último ejercicio, a manera de justificar la monocentricidad que se estaría desarrollando al interior de cada espacio regional, fue analizar los grados de policentrismo de manera particular para cada región, tomando en cuenta las ciudades seleccionadas en la metodología.

\section{Cuadro $\mathrm{N}^{\circ} 6$}

Saldos de la supremacía en población de Temuco sobre Angol

\begin{tabular}{|l|r|r|r|r|r|r|}
\hline Ciudades/Periodo & $1940-1952$ & $1952-1960$ & $1960-1970$ & $1970-1982$ & $1982-1992$ & $1992-2002$ \\
\hline Temuco-Angol & 0,21 & 0,27 & 0,88 & 0,38 & 0,31 & 0,69 \\
\hline
\end{tabular}

Fuente: Elaboración propia a partir de datos obtenidos de Zamudio, 1999 y INE, 2005.

Figura $\mathrm{N}^{\circ} 3$

Comparación de jerarquías urbanas entre 1940 y 2002

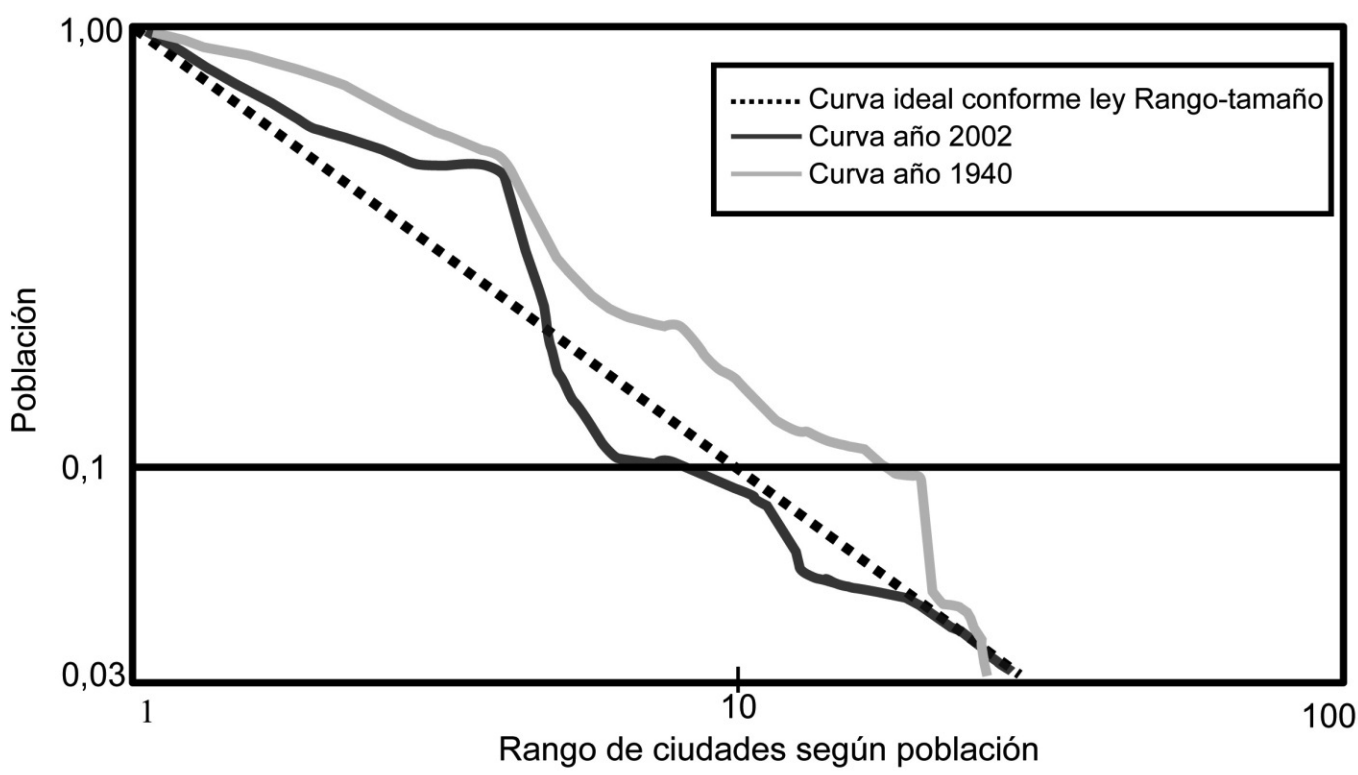

Fuente: Elaboración propia a partir de datos obtenidos de Zamudio, 1999 y INE, 2005. 
Cuadro $\mathrm{N}^{\circ} 7$

Medición de policentrismo regiones de La Araucanía, Los Ríos y Los Lagos

\begin{tabular}{|l|r|r|r|r|r|r|r|r|}
\hline Región de & \multicolumn{1}{|l|}{ Lsum } & Lmax & \multicolumn{1}{|l}{$\begin{array}{l}\text { Densidad } \\
\text { del grafo }\end{array}$} & \multicolumn{1}{l}{$\begin{array}{l}\text { Desv. grado } \\
\text { entrada }\end{array}$} & \multicolumn{1}{l}{$\begin{array}{l}\text { Desv. grado } \\
\text { salida }\end{array}$} & \multicolumn{1}{l}{$\begin{array}{l}\text { Psf } \\
\text { entrada }\end{array}$} & Psf salida & Pgf \\
\hline La Araucanía & 12.167 & 29.571 & 0,411 & 1450,090 & 646,282 & 0,214 & 0,221 & 0,218 \\
\hline Los Ríos & 1.976 & 3.761 & 0,525 & 181,542 & 167,703 & 0,228 & 0,233 & 0,230 \\
\hline Los Lagos & 8.501 & 19.186 & 0,443 & $1.016,424$ & 643,578 & 0,198 & 0,160 & 0,179 \\
\hline
\end{tabular}

Fuente: Elaboración propia.

Como se aprecia en el Cuadro $\mathrm{N}^{0} 7$, las tres regiones presentan valores de policentrismo muy bajos. Así, en la Región de La Araucanía nos encontramos con un grado de policentricidad de 0,218 , lo cual es esperable debido al efecto que desarrolla la ciudad de Temuco en su sistema. La densidad del grafo es la más baja de las tres regiones, lo que explica en cierta medida que el grado de policentrismo de la Región de La Araucanía haya sido mayor que el de la Región de Los Lagos (de valor 0,179). Además, la Región de La Araucanía presenta una homogeneidad de los flujos levemente superior.

La Región de Los Ríos es la que obtiene el valor de policentrismo más elevado, esto es 0,230 , posiblemente debido a las relaciones entre la desviación estándar del grado de entrada y grado de salida, ya que estos son levemente más homogéneos que los de las otras dos regiones, además con una densidad del grafo más elevada. A pesar de estas dos características, el grado de concentración de flujos en la ciudad de Valdivia estaría indicado el bajo nivel de policentrismo.

La Región de Los Lagos presentó el menor valor de policentrismo, posiblemente asociado a la existencia de dos centros mayores como Puerto Montt y Osorno, donde el primero controla la mayor parte de flujos de la red. El sistema de la isla de Chiloé también podría friccionar de cierta manera los flujos en el sistema, cuyas ciudades podrían seguir un patrón similar asociado posiblemente solo a la capital regional. Los valores de la desviación estándar (especialmente de entrada) muestran la menor homogeneidad que se desarrolla en Los Lagos respecto de las otras dos regiones. Es interesante destacar el bajo valor de policentrismo obtenido en la Región de los Lagos, pese al desarrollo salmonero que se da en la zona y a la relación de movilidad que se constata. Al parecer, la organización desarrollada por los centros urbanos de la zona no ha sido estructurada bajo un concepto de redes de ciudades, al estilo de Batten (1995), o en el concepto de réseaux de villes en Francia, donde los centros urbanos cooperan entre sí. Una publicación de Fløysand, Barton y Román (2010), muestra como la estructuración de la actividad salmonera no ha seguido una articulación coherente entre los centros urbanos en la isla Grande de Chiloé y las municipalidades no tendrían mayor injerencia en las decisiones que organizan y afectan su propio territorio.

Así, el resultado de policentrismo en las tres regiones y el análisis de la matriz dejan de manifiesto que la mayoría de los vínculos se realizan al interior del espacio regional. En efecto, muestran que si se analiza el grado de cohesión considerando el sistema en su conjunto, este estaría ocultando el fenómeno que realmente se desarrolla al interior de los espacios regionales.

Así, el grado de policentricidad de las estructuras urbanas de cada región aparece fuertemente polarizado, con un futuro difuso. En efecto, si se analiza la tendencia que han mostrado las disparidades entre centros urbanos, sería posible apreciar en la Región de La Araucanía el caso de Temuco con el mencionado agudizamiento de la disparidad en su espacio regional, respecto a la ciudad que le sigue (Angol), pasando de un 3,3 veces en 1940 a 6,1 veces para el año 2002. Para el caso de Valdivia, en la Región de Los Ríos, si bien el aumento no es considerable, pasa de 
ser un 4,6 veces superior al centro urbano de La Unión en 1940, a 5,0 el año 2002. En el caso de la Región de Los Lagos, Puerto Montt pasó de 4,4 a 5,3 veces el año 2002.

Por lo anterior, la ausencia de una política eficaz de ordenamiento territorial se refleja en los resultados obtenidos, e interpela a los planificadores hacer esfuerzos en materia de disminución de las disparidades regionales y a revisar y verificar los instrumentos que están siendo aplicados. En este sentido, si no hay una intervención explícita, se prevé un reforzamiento de la trayectoria actual del sistema, lo que se traducirá en mayores dificultades para la población que reside en sus centros urbanos, producto de la baja cohesión del mismo.

\section{Conclusiones}

Aunque el policentrismo es un concepto que no está del todo consolidado, resulta útil medirlo y analizarlo, tal como lo demuestran las numerosas investigaciones que han ido en esta dirección. En Chile, este concepto casi no ha sido tratado, por lo que este trabajo puede representar una contribución en cuanto a su comprensión en el territorio chileno. Además, el método presentado y las mejoras que se han propuesto pueden constituir importantes herramientas para comprender de mejor forma la estructura de un territorio, más aún si se piensa que existen mayores potencialidades que no han sido tratadas en este trabajo, como el análisis del policentrismo en el tiempo, después de una política territorial, para lo cual sería necesario contar con flujos en dos o más periodos de tiempo (Green, 2007; Ruiz y Gil-Mendieta, 2004).

Es necesario señalar que a pesar de lo rescatable que puede ser este tipo de análisis, los enfoques aquí planteados requerirían ser complementados. En efecto, los estudios de medición del policentrismo se han generado más bien desde una de las dos corrientes (morfológica o funcional), sin tomarlas en conjunto. Una publicación exploratoria que sigue esta línea es la planteada por Burger y Meijers (2010), que destaca la necesidad de aunar los dos enfoques para una mejor compresión de la jerarquía urbana.
El análisis del sistema de ciudades del sur de Chile considerado en este trabajo, dejó de manifiesto que los vínculos entre centros urbanos están más bien circunscritos al interior de su espacio regional, por lo que resultó más idóneo analizar qué sucedía al interior de estos espacios. En efecto, los resultados obtenidos sugieren la existencia de una tendencia monocéntrica en las regiones analizadas, ya que los valores de policentrismo obtenidos señalan una correcta interpretación de los fenómenos que afectan los territorios estudiados. Sin embargo, se considera que sería necesario complementar este trabajo con otro tipo de análisis, para poder reafirmar procesos de concentración y dependencia respecto de las capitales regionales, las que podrían estar reproduciendo el patrón monocéntrico sobre sus regiones, tal como lo ejerce la ciudad de Santiago a escala nacional, y que se traduce -según la DATAR (2010)- en una débil cohesión social y territorial y en todas las consecuencias nefastas que esto trae consigo para los habitantes de estos territorios.

Finalmente, se considera fundamental la revisión de los instrumentos de planificación territorial aplicados en el espacio regional, de tal forma de contar con herramientas capaces de ir en la dirección de disminuir las disparidades espaciales presentes. A la fecha, se constató que los instrumentos no han sido del todo eficaces, aún más, existe una profundización de las características monocéntricas al interior de cada espacio regional, generado por la baja cohesión territorial de las ciudades que lo componen.

\section{Referencias bibliográficas}

AGUILERA, A. Growth in commuting distances in French polycentric Metropolitan areas: Paris, Lyon and Marseille. Urban Studies, 2005, Vol. 42, № 9, p. 1537-1547.

BATTEN, D. Network Cities: Creative Urban Agglomerations for the 21st Century. Urban Studies, 1995, Vol. 32, No 2, p. 313327.

BAUDELLE, G. \& MONTABONE, B. Les échelles de la cohésion spatiale en Europe. In: Conference Culture, Cohesion and Competitiveness: Regional Perspectives 
European. Liverpool: Regional Science Association (ERSA), 27-31 Agosto, 2008.

BAUDELLE, G. \& PEYRONY, J. Le polycentrisme en France: cheminement d'un concept. Territoires 2030 Analyses et débats (DATAR), 2005, № 1, p. 89-101.

BECERRIL-PADUA, M. Policentrismo en las ciudades latinoamericanas. El caso de Santiago de Chile. Revista Theomai, 2000, № 1, p. 1-23.

BURGER, M. \& MEIJERS, E. Form Follows Function? Linking Morphological and Functional Polycentricity. GaWC Research Bulletin, 2010, № 344.

CAMAGNI, R. Economía urbana. Barcelona: Antoni Bosch editor, 2005.

CARRIÈRE, J.P. Une réflexion sur la construction du polycentrisme en Europe: apports et limites du rapport Potentials for polycentric development in Europe. Territoires 2030 Analyses et débats (DATAR), 2005, p. 47-64.

COLL, J. L. y ARENAS, F. Policentrismo y organización territorial: una lectura a partir del caso europeo. Revista Geográfica de Valparaíso, 2005, № 36, p 73-84.

CONSEJO DE RECTORES DE LAS UNIVERSIDADES CHILENAS. Anuario Estadístico 2008. Santiago de Chile: Secretaría General del H. Consejo de Rectores de las Universidades Chilenas, 2009.

D A TAR DÉLÉGATION À L'AMÉNAGEMENT DU TERRITOIRE ET À L'ACTION RÉGIONALE. La cohésion territoriale en Europe. Paris: La Documentation Française, 2010.

DAUPHINÉ, A. Les réseaux urbains: un exemple d'application de la théorie des systèmes auto organisés critiques. Annales de Géographie, 2003, Vol. Mai-Juin, № 631, p. 227-242.

DAVOUDI, S. Polycentricity: Panacea or pipedream?. En: CATTAN, N. (editor). Cities and networks in Europe: a critical approach of polycentrism. Southport: John Libbey Eurotext, 2007, p. 65-73.

DEMATTEIS, G. Globalisation and regional integration: the case of the Italian urban system. Geolournal, 1997, Vol. 43, No 4, p. 331-338.

ESPON. Potentials for polycentric development in Europe. Luxembourg: European Observation Network, Territorial Development and Cohesion ESPON 1.1.1, 2005.

EU EUROPEAN COMMUNITIES. European Spatial Development Perspective Towards Balanced and Sustainable Development of the Territory of the European Union, ESDP. Committee on Spatial Development, European Commission, 1999.

FLØYSAND, A., BARTON, J. y ROMÁN, A. La doble jerarquía del desarrollo económico y gobierno local en Chile: El caso de la salmonicultura y los municipios chilotes. EURE, 2010, Vol. 36, No 108, p. 123-148.

GLØERSEN, E. Faut-il des visions spatiales pour construire une Europe polycentrique? Territoires 2030 Analyses et débats (DATAR), 2005, p. 65-88.

GREEN, N. Functional Polycentricity: A Formal Definition in Terms of Social Network Analysis. Urban Studies, 2007, Vol. 44, № 11, p. 2077-2103.

GREEN, N. City-states and the spatial in between. Town \& Country Planning, 2008, $\mathrm{N}^{\circ}$ May, p. 224-231.

HALBERT, L.; PAIN, K. \& THIERSTEIN, A. European Polycentricity and emerging Mega -City-Regions- "one size fits all" policy? Built Environment, 2006, Vol. 32, № 2, p. 194-218.

HALL, P. \& PAIN, K. The polycentric Metropolis. Learning from mega-city regions in Europe. London: Earthscan, 2006.

HALL, P.; PAIN, K. \& GREEN, N. Anatomy of the Polycentric Metropolis: Eight MegaCity Regions in Overview. In: HALL, P. \& 
PAIN, K. The polycentric Metropolis. Learning from mega-city regions in Europe. London: Earthscan, 2006, p. 19-52.

HIDALGO, R.; DE MATTOS, C. y ARENAS, F. (editores). Chile: del país urbano al país metropolitano. Santiago de Chile: Serie GEOlibros 12, Instituto de Geografía y Colección EURE Libros Instituto de Estudios Urbanos y Territoriales, Pontificia Universidad Católica de Chile, 2009.

HIDALGO, R. y ARENAS, F. Del país urbano al país metropolitano. Transformaciones recientes en las ciudades chilenas. En: HIDALGO, R.; DE MATTOS, C. y ARENAS, F. (editores). Chile: del país urbano al país metropolitano. Santiago de Chile: Serie GEOlibros 12, Instituto de Geografía y Colección EURE Libros Instituto de Estudios Urbanos y Territoriales, Pontificia Universidad Católica de Chile, 2009. p. 9-29.

HORNIS, W. \& VAN ECK, J.R. A typology of peri-urban areas in the Netherlands. Tijdschrift voor Economische en Sociale Geografie, 2008, Vol. 99, № 5, p. 619-628.

INSTITUTO NACIONAL DE ESTADÍSTICAS DE CHILE (INE). Base censal redatam a nivel nacional. Santiago de Chile: INE, 2002.

INSTITUTO NACIONAL DE ESTADÍSTICAS DE CHILE (INE). Chile: Ciudades, Pueblos, Aldeas y Caseríos. Santiago de Chile: Instituto Nacional de Estadísticas, 2005.

LIMTANAKOOL, N.; DIJST, M. \& SCHWANEN, T. A Theoretical Framework and Methodology for Characterising National Urban Systems on the Basis of Flows of People: Empirical Evidence for France and Germany. Urban Studies, 2007, Vol. 44, № 11 , p. 2123-2145.

MATURANA, F. y CORNEJO, F. El estudio de sistemas de ciudades. Una aproximación desde métodos de análisis de redes. Tiempo y Espacio, 2010, № 25, p. 41-57.

MATURANA, F. y TERRA, P. La notion de ville moyenne au Chili, entre la fonctionnalité et la taille de population. In: Colloque International Villes petites et moyennes, un regard renouvelé, 9 y 10 de diciembre, 2010.
MEIJERS, E. Measuring Polycentricity and its Promises. European Planning Studies, 2008, Vol. 16, № 9, p. 1313-1323.

MICHEL, M. Ville moyenne, ville-moyen. Annales de géographie, 1977, Vol. 86, № 478, p. 641-685.

MINISTERIO DE EDUCACIÓN DE CHILE. Tabla de Matrícula Año 2009. Santiago de Chile: MINEDUC, 2010. Disponible en Internet: http://w3app.mineduc.cl/DedPublico/ archivos_de_datos

PARR, J. The Polycentric Urban Region: A Closer Inspection. Regional Studies, 2004, Vol. 38, No 3, p. 231-240.

PEÑA, F. y ESCALONA, M. Expansión urbana en la intercomuna Araucanía Centro. Alteraciones sobre las áreas rurales. En: HIDALGO, R.; DE MATTOS, C. y ARENAS, F. (editores). Chile: del país urbano al país metropolitano. Santiago de Chile: Serie GEOlibros 12, Instituto de Geografía y Colección EURE Libros Instituto de Estudios Urbanos y Territoriales, Pontificia Universidad Católica de Chile, 2009, p. 389-398.

POLYNET. POLYNET Action 1.1 Commuting \& the definition of functional urban regions. Dublin: Institute of Community Studies/The Young Foundation \& Polynet Partners, 2005.

PUMAIN, D. \& SAINT-JULIEN, T. Les interactions spatiales. Paris : Armand Colin, 2001.

RIGUELLE, F.; THOMAS, I. \& VERHETSEL, A. Measuring urban polycentrism: a European case study and its implications. Journal of Economic Geography, 2007, Vol. 7, № 2, p. 193-215.

ROJAS, C.; MUÑIZ, O. y GARCÍA-LOPEZ, $M$. Estructura urbana y policentrismo en el Área Metropolitana de Concepción. EURE, 2009, Vol. 35, No 105, p. 47-70.

ROZENBLAT, C. Tissus de villes. Réseaux et systèmes urbains en Europe, Habilitation à diriger des recherches. Université Montpellier III, 2004. 
RUIZ, A. y GIL-MENDIETA, J. Redes variables en el tiempo: visualización con Pajek. Redes. Revista hispana para el análisis de redes sociales, 2004, Vol. 6, № 4, p. 1-16.

VARELA, C. La interacción entre ciudades $y$ el ordenamiento territorial. Estudio de caso: Temuco (IX Región de La Araucanía) y Valdivia (Región de Los lagos), Chile. Revista Austral de Ciencias Sociales, 2001, № 5, p. 61-71.
WASSERMAN, S. \& FAUST, K. Social Network Analysis: Methods and Applications. Structural Analysis in the Social Sciences. New York: Cambridge University Press, 1994.

YUE, W.; LIU, Y. \& FAN, P. Polycentric urban development: the case of Hangzhou. Environment and Planning, 2010, Vol. 42, No 3, p. 563-577.

ZAMUDIO, O. Población de los centros poblados de Chile: 1875-1992. Santiago de Chile: INE, 1999. 